\title{
Medullary control of nociceptive transmission: Reciprocal dual communication with the spinal cord
}

\author{
Armando Almeida ${ }^{1, *}$, Hugo Leite-Almeida', Isaura Tavares ${ }^{2}$ \\ 'Life and Health Sciences Research Institute (ICVS), School of Health Sciences, University of Minho, 47I0-057 Braga, Portugal \\ ${ }^{2}$ Institute of Histology and Embryology, Faculty of Medicine, University of Oporto, Porto, Portugal
}

Control of pain perception, essential for organism surviving and recovery from disease, is exerted by higher brain centers integrating nociception with emotional and cognitive information and modulating the brainstem-spinal feedback loops that regulate spinal nociceptive transmission. Development of chronic pain deregulates the forebrain-brainstem-spinal pain control system, which leads to neuroplasticity and disruption of a balanced brain-spinal communication. Targets for impeding pain chronification are being developed using the manipulation of the cross talk between brain and dorsal horn, at both sites of the loop.

\section{Introduction}

In 'normal' conditions, pain triggered by a small lesion is less intense and disappears in shorter time than after a large lesion, which represents the expression of a major biological protection mechanism to preserve organism integrity [1]. This proportionality between lesion and pain can be modified during intense emotional and cognitive situations [2]. Moreover, pain associated to diseases has the biological protective function of restricting body activities and concentrating organism efforts on body healing. These adaptative conditions result largely from brain modulation of spinal nociceptive transmission [3,4], an endogenous

*Corresponding author: A. Almeida (aalmeida@ecsaude.uminho.pt)

\section{Section Editors:}

Frank Porreca - University of Arizona, Tucson, USA Michael Ossipov - University of Arizona, Tucson, USA

mechanism that can be modulated by reciprocal communications between the вRAINSTEM (see glossary) and spinal cord [4-8]. However, a prolonged pain condition can induce brain and spinal neuroplasticity associated to CENTRAL SENSITIZATION (see glossary) $[9,10]$. The resulting changes in neuronal properties can lead to a deregulation of the brain mechanisms modulating pain, which may account for the maintenance of CHRONIC PAIN (see glossary) long after lesion healing.

\section{Endogenous pain control system}

The PAG-RVM-dorsal horn circuitry

Although changes in pain behavior have been demonstrated by manipulation of several brain areas, the main pain control centers are located in the brainstem. The mesencephalic periaqueductal grey (PAG) and the rostral ventromedial medulla (RVM) are known for long to be implicated in endogenous pain control through an antinociceptive PAG-RVM-dorsal horn descending circuitry [11] (Fig. 1a). However, increasing evidences also implicate the RVM in descending facilitation of spinal nociceptive transmission $[9,11]$. This dual effect is correlated with the co-existence at the RVM of neurons involved in descending antinociceptive actions (OFF-cells), along with cells responsible for descending pronociceptive effects (ON-cells) (Fig. 1a). 


\section{Glossary}

Allodynia: pain due to a stimulus that does not normally provoke pain Brainstem: the portion of the brain consisting of the medulla oblongata, pons and mesencephalon. It is the major route for communication between the forebrain and the spinal cord and controls various functions including respiration and regulation of heart rhythms. It includes the part of the reticular formation that plays a central role in bodily and behavioral alertness.

Central sensitization: increased excitability of central paintransmitting neurons that may result from prolonged nociceptive barrage and involves descending nociceptive modulation from the brain. Chronic pain: pain that persists beyond resolution of an underlying disorder or healing of an injury, and that is often more intense than the underlying process would predict.

Forebrain: the most anterior segment of the adult brain that develops from the embryonic telencephalon and diencephalon and includes the cerebrum (cortex, hippocampus, amygdala), thalamus and hypothalamus.

Hyperalgesia: an increased response to a stimulus that is normally painful.

Limbic system: a group of interconnected deep brain structures common to all mammals that includes the hypothalamus, amygdala, olfactory bubs and hippocampus. It plays an important role in the regulation of motivation, emotions and various autonomic functions. Neuropathic pain: pain initiated or caused by a primary lesion or dysfunction in the nervous system.

NKI receptors: receptors for ligation of substance $P$, one of the main neurotransmitters released by primary afferent nociceptive fibers in the spinal dorsal horn.

NMDA receptors: subtype of Glutamate receptors involved in synaptic potentiation at several neural pathways, including the pain system. Their activation at spinal synapses leads to facilitation of postsynaptic responses and enhanced neurotransmitter release and is implicated in central sensitization and chronic pain mechanisms.

Peripheral sensitization: increased excitability of peripheral nociceptors (primary afferents), often mediated by an inflammatory process.

\section{Forebrain modulation of pain: role of the brainstem}

Pain is a multidimensional experience where the sensorydiscriminative information conveyed from the periphery by the nociceptive system is integrated with the emotional environment processed by the amygdala (AMY) and cognitive information concerning past experiences specific for each individual [12]. The motivational-affective component of the pain experience suggests that LIMBIC areas (see glossary) like the AMY, the insular cortex (INS) and the anterior cingulated cortex (ACC) should have the capacity to regulate the endogenous pain control system and the final pain perception. These FOREBRAIN areas (see glossary) are highly interconnected and output nuclei have neurons with long projecting axons terminating in areas like the PAG and RVM (Fig. 1). Several areas of the caudal medulla oblongata involved in pain modulation like the dorsal reticular nucleus (DRt), the caudal ventrolateral medulla (CVLM) and the nucleus tractus solitarius (NTS) are also targeted by the forebrain, which indicates that emotions and cognition can directly control brainstem areas implicated in nociceptive modulation (Fig. 1). Accordingly, depending on environmental and emotional conditions, the AMY, INS and ACC may exert a dual facilitating and inhibiting role in pain modulation $[13,14]$ mediated by caudal brainstem areas, such as the RVM and DRt [13].

\section{The caudal medulla oblongata}

The caudalmost portion of the brainstem has several areas involved in pain modulation. Besides the DRt $[3,15,16]$, CVLM [6] and NTS [17] referred above, it arbors other pain control centers like the ventral reticular [16] and paratrigeminal nuclei [18]. Like the RVM, the caudal medulla oblongata exerts a dual descending modulatory action by inhibiting or increasing nociception. Thus, the caudal medulla oblongata contributes to the fine balance between facilitation and inhibition of spinal nociceptive transmission and, ultimately, to pain perception. However, the descending modulatory actions exerted by the DRt and the CVLM exhibit special characteristics because they are conveyed by reciprocal monosynaptic spino-bulbo-spinal loops. Besides allowing rapid and dynamic pain modulation, these feedback loops may allow that sudden changes of the nociceptive homeostasis triggers an adequate response of both medullary and spinal nociceptive neurons. On the basis of the extensive characterization of the brain connections of the DRt and CVLM, it is likely that the brainstem-spinal reciprocal loops also integrate nociception with other types of physiological information arriving from the periphery and important for body homeostasis.

\section{The dorsal reticular nucleus}

The brain-DRt-spinal circuitry is now well characterized $[3,19]$ and several studies indicate that the DRt is implicated in nociceptive processing and modulation (Fig. 2a) [3,15]: DRt neurons are exclusively or mainly activated by cutaneous or visceral noxious stimulation; the DRt receives projections from spinal dorsal horn nociceptive neurons that establish asymmetrical, potentially excitatory, synaptic contacts with DRt neurons projecting back to the dorsal horn [3]; DRt neurons project to the dorsal horn and modulate spinal nociceptive activation [16] by establishing asymmetrical synapses with spinal neurons projecting back to the DRt. These data indicate that a reciprocal excitatory feedback loop occurs between the DRt and spinal dorsal horn, through which ascending nociception is increased by the descending branch of the circuit [3]. Moreover, DRt neurons project to [19] and receive projections from [3] brain areas implicated in the direct or indirect control of spinal nociceptive transmission, such as the CVLM, NTS, PAG, RVM, AMY, locus coeruleus (noradrenergic antinociceptive centre) and paraventricular hypothalamic nucleus (PVN - the main effector of the physiological response to stress). This suggests that reciprocal feedback loops occur not only between the DRt and the spinal cord but also between the DRt and brainstem and forebrain 
areas modulating pain. Finally, DRt neurons project simultaneously to the spinal dorsal horn and the medial thalamus, an area that allows direct access to the neocortex [20]. This indicates that balanced nociception achieved by the DRtspinal cord interaction can reach forebrain areas implicated in the affective dimension of pain.

The extensive ascending and descending connections with forebrain, brainstem and spinal areas implicated in pain processing and modulation supports the pain facilitating $[3,16]$ and pain inhibiting [15] actions attributed to the DRt (Fig. 2a), as well as its role as a relay station mediating pain modulation triggered by the neocortex [13].

\section{The caudal ventrolateral medulla}

The characterization of reciprocal connections between the CVLM and the spinal cord suggested a special role of its lateralmost part- the VLMlat- in pain modulation [6]. The VLMlat targets exclusively spinal layers involved in nociceptive transmission, namely laminae I, IV-V and $\mathrm{X}$, and receives nociceptive input from laminae I-III [6]. That the VLMlat is the CVLM region more relevant in pain modulation is in agreement with data showing that the hypoalgesia induced following VLMlat stimulation is more pronounced and long lasting than from other CVLM regions [6]. A closed reciprocal loop connects the VLMlat with lamina I (Fig. 2b) and the synaptic characterization of the loop suggests that the ascending nociceptive input triggers descending modulation mainly by inhibitory mechanisms and, in a lower extension, facilitatory (see glossary) actions [6]. These observations postulated that the CVLM could also be involved in pronociceptive effects, which was recently supported by the demonstration of coexistence of $\mathrm{ON}$ and OFF cells in the CVLM [21]. The functional relevance of the spino-VLMlatspinal loop was demonstrated by the differential involvement of different types of lamina I neurons projecting to the VLMlat and by different nociceptive activation of these neuronal types after VLMlat lesions [6].

Descending modulation from the CVLM is dynamically conveyed by direct and indirect complex circuits. The $\alpha_{2}$-adrenoreceptor mediated inhibition of spinal nociceptive transmission exerted by the CVLM is conveyed by the pontine $\mathrm{A}_{5}$ noradrenergic cell group, whereas descending serotonergic modulation appears to involve the RVM [6]. Besides the $A_{5}$ noradrenergic cell group and the RVM, other brain regions are probably recruited in the descending modulation from the CVLM because this area targets pain control centers like the PAG, parabraquial area and locus coeruleus (Fig. 2b). The pattern of CVLM connections in the brain further suggests that it may play an important role in the integration of pain responses with other body functions. The CVLM participates in pain-cardiovascular integration and in the hypoalgesia detected during hypertensive conditions [22,23]. Finally, the arrival of spinal nociceptive input to the CVLM is also integrated with the information derived from widespread brain areas [24]. The CVLM receives strong input from areas participating in somatosensory processing, and autonomic and motor controls. More than just providing a mechanism of feedback integration, the reciprocal spinoCVLM loop is likely to play a complex role on the protective homeostatic function of the pain system.

\section{Chronic pain and the brainstem-spinal dorsal horn communication}

Importance of controlling nociceptive input to the brain

Acute pain and transient ALLODYNIA and HYPERALGESIA (see glossary) associated to inflammation, chronic pain and disease are crucial examples of the importance of pain for organism protection (Fig. 1). Conversely, other situations require analgesia to preserve organism integrity and surviving, which indicates that pain suppression is as important to the organism as pain perception [2]. Fear and acute stress, for example, strongly inhibit pain as it could be incompatible with the need of running from eminent danger. Accordingly, the AMY and the PVN, areas responsible for emotional processing of fear and initiation of the physiological response to stress, respectively, have shown to modulate pain $[25,26]$.

Recent data indicate that the analgesic action triggered by PVN stimulation changes the activity of $\mathrm{ON}$ and OFF cells also present in the RVM and CVLM [21,27], which is exerted through a descending serotonergic and noradrenergic pathway inhibiting spinal nociceptive neurons (unpublished). These PVN actions upon the medulla oblongata and spinal cord are paralleled by a correspondent inhibition of pain-like behavior [21,27]. The AMY exerts also a fine control upon RVM ON and OFF cell activities, and the data collected indicate that the AMY is also implicated in pain facilitation $[26,28]$. Finally, the ACC, a key area in the processing of the motivational-affective dimension of pain was also shown to have a descending nociceptive facilitating action, which is mediated by two relay medullary centers, the RVM and the DRt [13] (Fig. 2a). All these data strongly implicate medullary pronociceptive and antinociceptive centers reciprocally connected with the spinal dorsal horn, as relay stations for the pain modulatory actions triggered by forebrain areas regulating pain experience. Importantly, the induction of chronic pain can change the modulatory action of the forebrain upon brainstem neurons and, consequently, upon and spinal neurons and nociceptive transmission (Fig. 1b). This was recently demonstrated for the PVN, as its actions upon RVM and CVLM ON and OFF cells and spinal convergent nociceptive neurons changed after the development of arthritis [21,27]. Thus, prolonged pain states are capable of changing the physiological homeostasis of the forebrainbrainstem-spinal cord neuronal network implicated in pain modulation. 
(a)

\section{Endogenous Pain Control System}

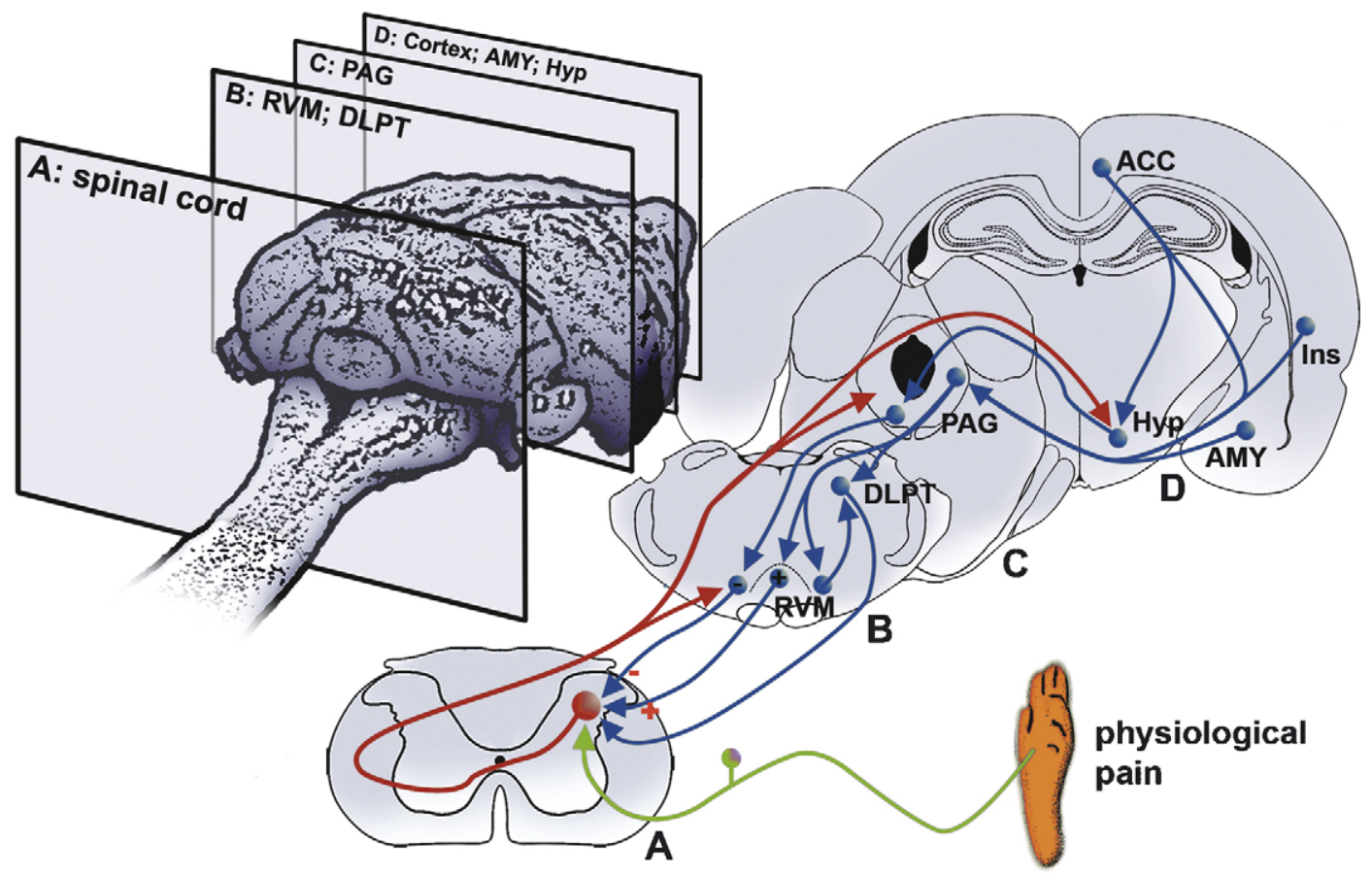

(b)

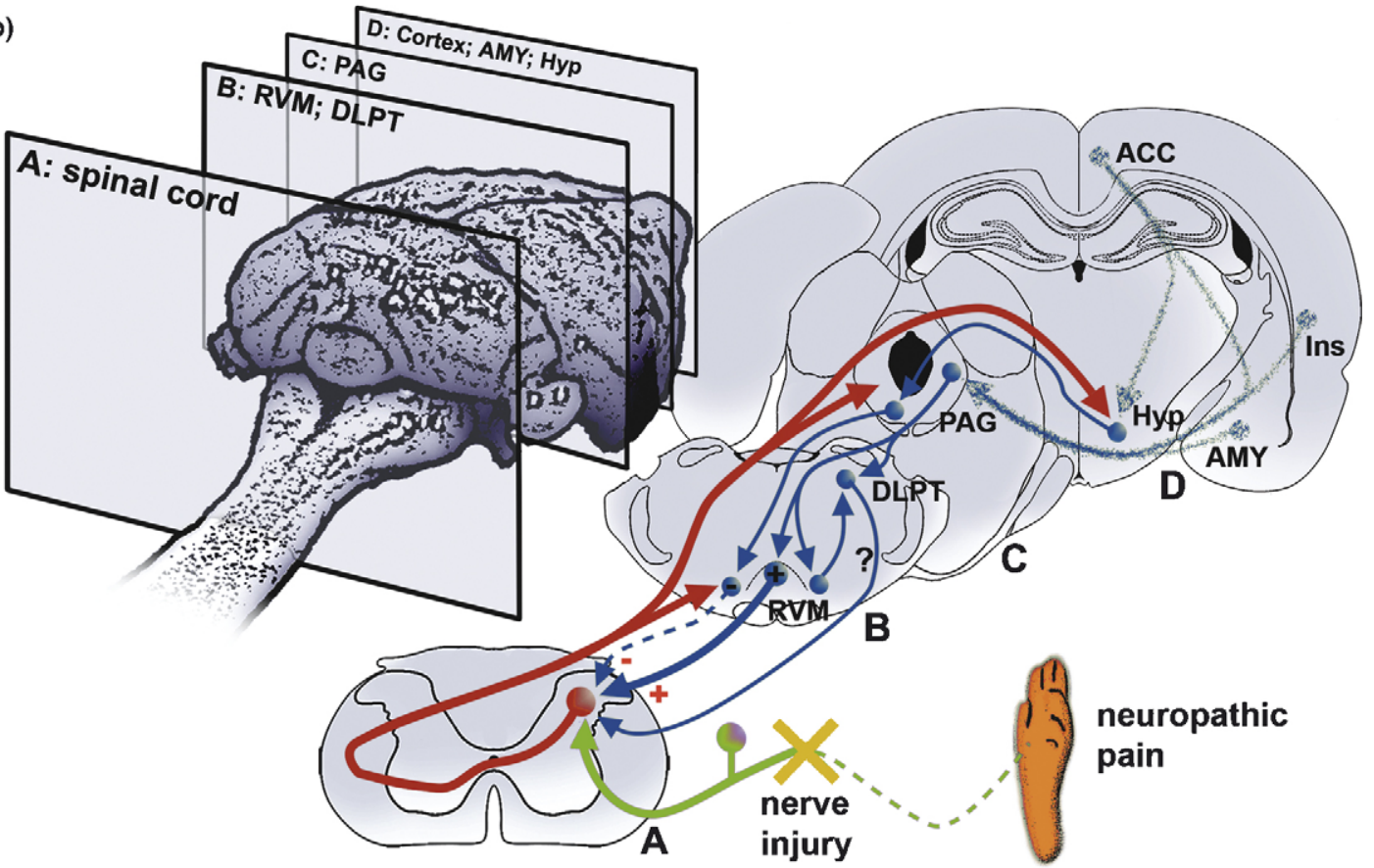

Figure I. Schematic representation of the supraspinal endogenous pain control system following physiological (a) and chronic (b) pain. Ascending pain pathways (red arrows) convey nociception to different targets along the brain, including the major brainstem pain modulating areas. Descending neuronal projections (blue arrows) between brain areas relevant for descending pain control extend from the forebrain to the spinal cord dorsal horn. Under physiological conditions (a) the RVM contains nociceptive neurons that project to the spinal dorsal horn and facilitate (+: ON-cells) or inhibit (-: OFF-cells) spinal nociceptive transmission arriving from peripheral nociceptors (green arrow). Pain chronification (b) resulting from peripheral (large green arrow) and central (large red arrows) sensitization induces changes in the endogenous pain control system, which may include disruption of the antinociceptive system (-: dashed blue arrow) and exacerbation of pronociceptive mechanisms (+: large blue arrow). Changes induced at forebrain levels are less known and are represented by blurred blue arrows. It should be noted that the RVM plays also a role in the noradrenergic descending control mediated by the locus coeruleus (DLPT). The three-dimensional rat brain profiles at the left of Ia and Ib represent the levels of the brain coronal sections A-D. A-D are central nervous system sections adapted with permission from the Paxinos and Watson Atlas [4I] and represent the spinal dorsal horn (A), the medulla oblongata and pons (B), the mesencephalon (C) and the 
Table I. Medullary-spinal circuitries: roles in pain perception and potential future therapies for chronic pain treatment

\begin{tabular}{|c|c|c|c|c|c|c|}
\hline $\begin{array}{l}\text { Brainstem-spinal } \\
\text { circuit }\end{array}$ & $\begin{array}{l}\text { Strategic approach } \\
\text { to the circuit }\end{array}$ & $\begin{array}{l}\text { Expected outcome } \\
\text { of intervention } \\
\text { at the circuit }\end{array}$ & $\begin{array}{l}\text { Who is } \\
\text { working in } \\
\text { the circuit }\end{array}$ & $\begin{array}{l}\text { Potential } \\
\text { therapies }\end{array}$ & $\begin{array}{l}\text { Advantages/ } \\
\text { disadvantages } \\
\text { of these therapies }\end{array}$ & Refs \\
\hline DRt-spinal & $\begin{array}{l}\text { - DRt chemical or } \\
\text { electrical stimulation } \\
\text { or inhibition } \\
\text { - DRt local } \\
\text { administration } \\
\text { of drugs }\end{array}$ & $\begin{array}{l}\text { Pronociception - } \\
\text { increased } \\
\text { (DRt stimulation) or } \\
\text { decreased } \\
\text { (DRt inhibition) } \\
\text { pain behaviour }\end{array}$ & $\begin{array}{l}\text { Almeida, A et al. } \\
{[3,16,19]} \\
\text { Tavares, I et al. } \\
{[33,35]} \\
\text { Villanueva et al. } \\
{[15,20]}\end{array}$ & $\begin{array}{l}\text { - Gene therapy } \\
\text { (GT) } \\
\text { - Intracerebral } \\
\text { lesioning (ICL) }\end{array}$ & $\begin{array}{l}\text { (GT): Induction of local } \\
\text { production of opioid } \\
\text { drugs/very preliminary } \\
\text { studies } \\
\text { (ICL): Potential block } \\
\text { of pronociception in } \\
\text { chronic pain processing/ } \\
\text { Location between two } \\
\text { respiratory areas }\end{array}$ & $\begin{array}{l}{[3,15,16} \\
19,20 \\
33,35]\end{array}$ \\
\hline ACC-DRt-spinal & $\begin{array}{l}\text { Stimulation/lesioning } \\
\text { the ACC and DRt, } \\
\text { respectively }\end{array}$ & $\begin{array}{l}\text { Pronociceptive } \\
\text { modulation mediated } \\
\text { by he limbic system }\end{array}$ & $\begin{array}{l}\text { Zhang, L et al. } \\
\text { [13] }\end{array}$ & $?$ & $?$ & {$[13]$} \\
\hline CVLM-spinal & $\begin{array}{l}\text { - Local chemical or } \\
\text { electrical stimulation } \\
\text { or inhibition } \\
\text { - Local administration } \\
\text { of drugs } \\
\text { - C-fos visualization of } \\
\text { nociceptive neurons }\end{array}$ & $\begin{array}{l}\text { Antinociception - } \\
\text { decreased } \\
\text { pain behaviour }\end{array}$ & $\begin{array}{l}\text { Tavares, I et al. } \\
{[6,22-24]} \\
\text { Pertovaara, A and } \\
\text { Almeida, A }[2 I]\end{array}$ & - Gene therapy & $\begin{array}{l}\text { Induction of opioid } \\
\text { production by CVLM- } \\
\text { spinal neurons/very } \\
\text { preliminary studies and } \\
\text { motor and respiratory } \\
\text { side effects }\end{array}$ & {$[6,21-24]$} \\
\hline NTS-spinal & $\begin{array}{l}\text { - NTS lesioning } \\
\text { - proinflammatory } \\
\text { cytokines }\end{array}$ & $\begin{array}{l}\text { Sickness-induced } \\
\text { hyperalgesia }\end{array}$ & $\begin{array}{l}\text { Watkins, LR and } \\
\text { Maier, SF [I7] }\end{array}$ & $?$ & $?$ & {$[17]$} \\
\hline RVM-spinal & $\begin{array}{l}\text { - Local administration } \\
\text { of drugs } \\
\text { - Destruction of substance } \\
\text { P spinal neurons } \\
\text { - Excitatory transmitter } \\
\text { release }\end{array}$ & $\begin{array}{l}\text { Pronociception and/or } \\
\text { Antinociception }\end{array}$ & $\begin{array}{l}\text { Porreca, F et al., } \\
{[5,9,32]} \\
\text { Dickenson, A, [7] } \\
\text { Fields, H, } \\
\text { Heinricher, M, [I I] } \\
\text { Dubner, R et al. } \\
\text { [3I] }\end{array}$ & $\begin{array}{l}\text { - Ablation of opioid } \\
\text { RVM cells } \\
\text { - Cholecystokinin } \\
\text { antagonists } \\
\text { - NMDA antagonists } \\
\text { - Serotonergic pain- } \\
\text { facilitating pathways }\end{array}$ & $\begin{array}{l}\text { - Reduction of neuropathic } \\
\text { pain/neuronal death } \\
\text { - Prevention of opioid } \\
\text { tolerance }\end{array}$ & $\begin{array}{l}{[5,7,9,11} \\
31,32]\end{array}$ \\
\hline AMY-RVM-spinal & $\begin{array}{l}\text { - Local administration } \\
\text { of opioids }\end{array}$ & $\begin{array}{l}\text { Antinociception } \\
\text { mediated by the } \\
\text { limbic system }\end{array}$ & $\begin{array}{l}\text { Heinricher et al. } \\
\text { [26] }\end{array}$ & $?$ & $?$ & [26] \\
\hline PVN-RVM-spinal & $\begin{array}{l}\text { - Local chemical stimulation } \\
\text { or inhibition } \\
\text { - Local administration } \\
\text { of drugs }\end{array}$ & $\begin{array}{l}\text { Stress-induced } \\
\text { analgesia }\end{array}$ & $\begin{array}{l}\text { Pertovaara, A and } \\
\text { Almeida, A [27] }\end{array}$ & $?$ & $?$ & {$[27]$} \\
\hline
\end{tabular}

Role of the brainstem in the development of chronic pain Chronic inflammatory or NEUROPATHIC PAIN (see glossary) results from prolonged nociceptive barrage to the central nervous system and the resulting neuroplasticity of peripheral and central components of the nociceptive system. Central and PERIPHERAL SENSITIZATION (see glossary) increase neuronal excitability along the ascending and descending pain pathways, which may disrupt the fine balance between pronociceptive and antinociceptive pain modulatory actions descending from the brain $[4,29]$. The reciprocal anatomical connections between the medulla oblongata and the spinal cord should play a role on neuronal plasticity of brainstem and spinal dorsal horn areas and in the impairment of pain behavior in chronic pain syndromes. Accordingly, the RVM and PAG are involved in human central sensitization [30], whereas in the rat the RVM is implicated in the maintenance of central sensitization in persistent inflammatory, neuropathic and visceral pain states [9,31] (Fig. 1b). The RVM is also responsible for the increased release of nociceptive neurotransmitters by peripheral nociceptors in the spinal dorsal

forebrain (diencephalon and telencephalon), (D). Abbreviations: ACC, anterior cingulate cortex; AMY, amygdala; CVLM. caudal ventrolateral medulla; DRt, dorsal reticular nucleus; Hyp, hypothalamus; DLPT, dorsolateral pontine tegmentum; Ins, insular cortex; PAG, periaqueductal gray matter; RVM, rostral ventromedial medulla. 


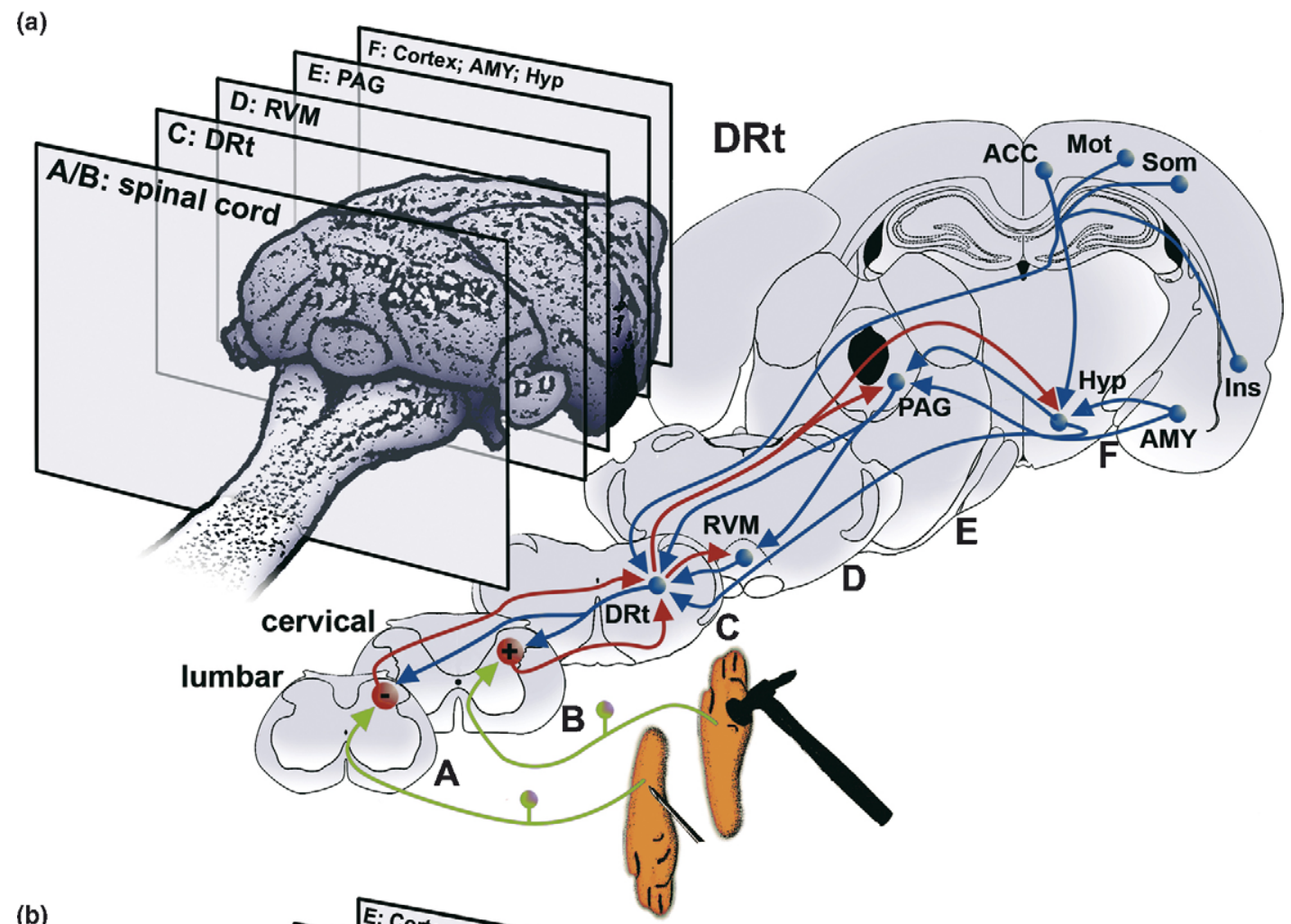

(b)

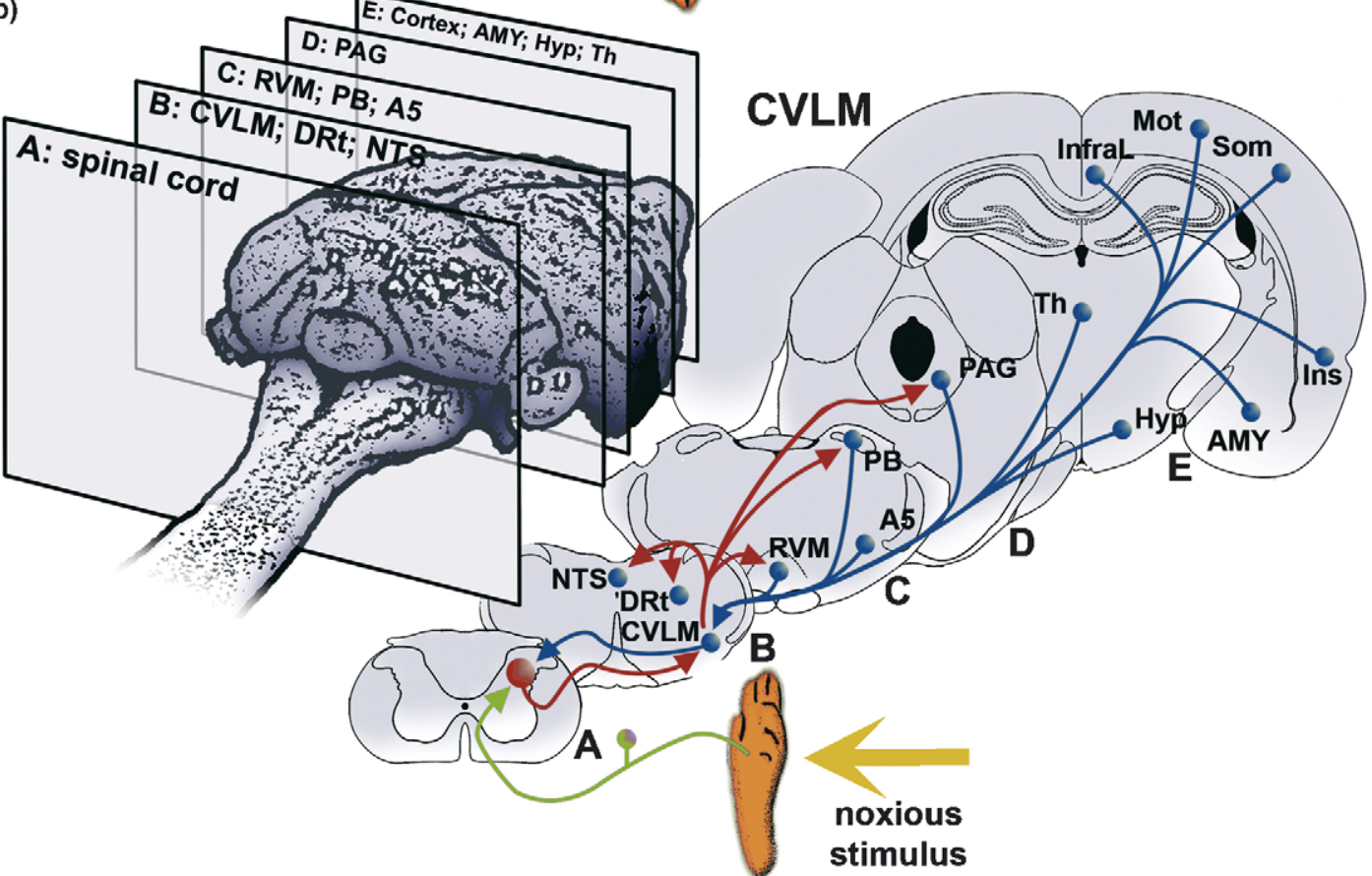

Figure 2. Spino-bulbo-spinal and brain neuronal network underlying the DRt (a) and the CVLM (b) involvement in pain modulating circuitries. In what concerns the DRt (a) note the reciprocal connections with the spinal dorsal horn, Hyp, PAG and RVM. The DRt has a pronociceptive action upon the spinal nociceptive transmission arriving from the front paw dermatome submitted to strong noxious stimulation ( + in the neuron located at the spinal level stimulated by hammering the forepaw) [3]. Moreover, it appears to mediate the inhibition of heterotopic nociception ( - in the neuron located at the spinal level stimulated by pricking the hindpaw dermatome), a phenomenon known as diffuse noxious inhibitory control (DNIC) (see reference [15] for details). The limbic system projects massively to the DRt. In what concerns the CVLM (b), note the large afferent projections from several cortical, subcortical and diencephalic areas of the brain and the reciprocal connections with the PAG, parabrachial nuclei, RVM and spinal cord. Ascending connections are represented in red, descending projections are in blue and nociceptors are depicted in green. A-D are central nervous system sections adapted with permission from the Paxinos and Watson Atlas [4I] and represent the spinal dorsal horn (A), the medulla oblongata and pons (B), the mesencephalon (C) and the forebrain (diencephalon and telencephalon, D). Abbreviations: A5, A5 noradrenergic cell group; ACC, anterior cingulate cortex; AMY, amygdala; CVLM. caudal ventrolateral medulla; DRt, dorsal reticular nucleus; Hyp, hypothalamus; Infral, limbic cortex; Ins, insular cortex; Mot, Motor cortex; NTS, nucleus tractus solitarius; PAG- periaqueductal gray matter; PB, parabrachial nuclei; RVM, rostral ventromedial medulla, Som, somatosensory cortex; Th, thalamus. 
horn during experimental neuropathy [32]. Moreover, superficial dorsal horn projection neurons expressing neurokinin-1 receptors (NK1-R) (see glossary), which are activated by excitatory neurotransmitters released by peripheral nociceptors, control spinal excitability and pain sensitivity through brainstem serotonergic facilitatory pathways descending along the dorsolateral funiculus $[7,8]$. Interestingly, the expression of NK1-R in spinal neurons projecting to brainstem areas involved in pain modulation differs with the target as the expression of NK1-R in the spino-CVLM pathway is stronger than that in the spino-DRt, which appears to exhibit mainly $\mathrm{GABA}_{\mathrm{B}}$ receptors [33].

The role of the two reciprocal bulbospinal loops centered at the DRt and VLMlat in inflammatory and chronic inflammatory pain is only beginning to be addressed. In both pain models the activity of the DRt was shown to be increased [34]. During arthritis, a strong decrease in the expression of $\mathrm{GABA}_{\mathrm{B}}$ and $\mu$-opioid receptors in DRt and CVLM nociceptive neurons was demonstrated ([35], unpublished), which might account for a lower efficacy of drugs acting upon these receptor systems. Accordingly, the intensity of nociceptive neuronal activation at the CVLM is negatively correlated with nociceptive activation of lamina I neurons (unpublished). Finally, we have recently demonstrated an involvement of both spinomedulllary loops in the differential expression of NK1 and $G_{A B A}$ receptors in spinal nociceptive neurons during secondary hyperalgesia induced by arthritic pain (unpublished). These data demonstrate the importance of controlling the cross talk between the brainstem and spinal cord in pain perception. Accordingly, the endogenous antinociceptive pain control system is disrupted in a dysfunctional pain syndrome like fibromyalgia and may account for the generalized hypersensitivity shown by these patients [10].

\section{Conclusions}

The brainstem concentrates all regions directly modulating the ascending nociceptive transmission from the spinal dorsal horn and mediates the pain control orders triggered by the forebrain areas responsible for coordination of the final pain perception. Moreover, the brainstem (and the medial thalamus) is also the main recipient of the spinal nociceptive information concerning the motivational-affective dimension of pain. This indicates that the brainstem and spinal cord are privileged areas for assuming the permanent homeostasis of the nociceptive system and its integration with other body functions through a continuous balance mediated by reciprocal feedback loops.

Can a better knowledge of the brainstem-spinal crosstalk be used in future therapies for pain control? On the basis of the extensive connections of pain control centers and on their involvement in several body functions, the search for neuronal specific targets of manipulation should be envisaged (Table 1). Gene therapy with vectors that target specific neurons and deliver therapeutic transgenes, like opioid precursors, in an inducible and sustained manner could be important in the future. The reciprocal bulbospinal loops implicated in pain control could be targeted by spinal administration of viral vectors that migrate to the caudal medulla. Thus, future therapies aimed at specifically increasing the serotonergic and noradrenergic descending control and the opioidergic, GABAergic and glycinergic 'pain' inhibiting action at the spinal level may prove effective in providing analgesia. On the contrary, neuroinflammatory factors involving not only neuronal networks but also glial cells and the immune system are actually under intense research in studies involving mainly chronic neuropathic pain models. However, no data are available on how the reciprocal networks between the brainstem and spinal cord are affected by the immune and glial changes accompanying prolonged inflammation and chronification of pain. Finally, a potential therapeutical target involving crosstalk between the brain and spinal cord could be the development of more specific drugs for modulating wind-up and long term potentiation (LTP) in spinal neurons following pain syndromes [36]. These two mechanisms induce increased/prolonged activity of nociceptive neurons/excitatory synapses and are implicated in central sensitization at both the brain and dorsal horn levels. Thus, they are both potentially present in supraspinally projecting spinal nociceptive neurons and nociceptive brainstem neurons modulating spinal nociceptive transmission. Reinforcing the necessity of exploring this hypothesis, endogenous spinal opioid receptors were shown to play a role in the endogenous spinal opioid mechanisms involved in windup [37], whereas GABA and NMDA RECEPTORS (see glossary) participate in spinal LTP induction and maintenance $[38,39]$. Future studies at the molecular level concerning the identification of specific characteristics of the pain neuronal network and improvement of membrane receptor cellular biology [40] are needed to develop well localized targeting strategies to control pain. However, although promising and exciting, the power of controlling the feedback interactions between the brain and spinal cord in terms of pain mechanisms is conceptually and technically complex and far from being achieved [38].

\section{Acknowledgements}

This work was supported by Science and Technology Foundation (FCT) Project POCTI/NSE/46399/2002 and FEDER and by Grünenthal Foundation (Portugal).

\section{References}

1 Wall, P.D. (2000) Pain: The Science of Suffering. Columbia University Press

2 McNally, G.P. (1999) Pain facilitatory circuits in the mammalian central nervous system: their behavioral significance and role in morphine analgesic tolerance. Neurosci. Biobehav. Rev. 23, 1059-1078 
3 Lima, D. and Almeida, A. (2002) The medullary dorsal reticular nucleus as a pronociceptive centre of the pain control system. Prog. Neurobiol. 66, 81108

4 Vanegas, H. and Schaible, H.G. (2004) Descending control of persistent pain: inhibitory or facilitatory? Brain Res. Brain. Res. Rev. 46, 295-309

5 Kovelowski, C.J. et al. (2000) Supraspinal cholecystokinin may drive tonic descending facilitation mechanisms to maintain neuropathic pain in the rat. Pain $87,265-273$

6 Tavares, I. and Lima, D. (2002) The caudal ventrolateral medulla as an important inhibitory modulator of pain transmission in the spinal cord. $J$. Pain 3, 337-346

7 Suzuki, R. et al. (2002) Superficial NK1-expressing neurons control spinal excitability through activation of descending pathways. Nat. Neurosci. 5, 1319-1326

8 Khasabov, S.G. et al. (2005) Spinal neurons that express NK-1 receptors modulate descending controls that project through the dorsolateral funiculus. J. Neurophysiol. 93, 998-1006

9 Porreca, F. et al. (2002) Chronic pain and medullary descending facilitation. Trends Neurosci. 25, 319-325

10 Julien, N. et al. (2005) Widespread pain in fibromyalgia is related to a deficit of endogenous pain inhibition. Pain 114, 295-302

11 Fields, H.L. et al. (2006) Central nervous system mechanisms of pain modulation. In Wall and Melzack's Textbook of Pain Online (McMahon, S.B. and Koltzenburg, M., eds), pp. 125-142, Elsevier

12 Price, D.D. (1999) Psychological mechanisms of pain and analgesia. IASP Press

13 Zhang, L. et al. (2005) Anterior cingulate cortex contributes to the descending facilitatory modulation of pain via dorsal reticular nucleus. Eur. J. Neurosci. 22, 1141-1148

14 Jasmin, L. et al. (2003) Analgesia and hyperalgesia from GABA-mediated modulation of the cerebral cortex. Nature 424, 316-320

15 Villanueva, L. et al. (1996) The medullary subnucleus reticularis dorsalis (SRD) as a key link in both the transmission and modulation of pain signals. Pain 67, 231-240

16 Almeida, A. et al. (1999) The medullary dorsal reticular nucleus facilitates pain behavior induced by formalin in the rat. Eur. J. Neurosci. 11, 110-122

17 Watkins, L.R. and Maier, S.F. (2000) The pain of being sick: implications of immune-to-brain communication for understanding pain. Annu. Rev. Psychol. 51, 29-57

18 Koepp, J. et al. (2006) Role of the paratrigeminal nucleus in nocifensive responses of rats to chemical, thermal and mechanical stimuli applied to the hind paw. Pain 122, 235-244

19 Leite-Almeida, H. et al. (2006) Brain projections from the medullary dorsal reticular nucleus: an anterograde and retrograde tracing study in the rat. Neuroscience 140, 577-595

20 Monconduit, L. et al. (2002) The integrative role of the rat medullary subnucleus reticularis dorsalis in nociception. Eur. J. Neurosci. 16, 937-944

21 Pinto-Ribeiro, F. et al. (2006) Descending pain modulatory influence from the hypothalamic paraventricular nucleus through the caudal ventrolateral medulla in arthritic and control rats. 5th FENS Forum, 4.13

22 Tavares, I. et al. (1997) Lesions of the caudal ventrolateral medulla block the hypertension-induced inhibition of noxious-evoked c-fos expression in the rat spinal cord. Eur. J. Pain 1, 149-160

23 Morato, M. et al. (2006) Inhibition of nociceptive responses of spinal cord neurones during hypertension involves the spinal GABAergic system and a pain modulatory center located at the caudal ventrolateral medulla. $J$. Neurosci. Res. 83, 647-655

24 Cobos, A. et al. (2003) Brain afferents to the lateral caudal ventrolateral medulla: a retrograde and anterograde tracing study in the rat. Neuroscience 120, 485-498

25 Yang, J. et al. (2006) Through the central V2, not V1 receptors influencing the endogenous opiate peptide system, arginine vasopressin, not oxytocin in the hypothalamic paraventricular nucleus involves in the antinociception in the rat. Brain Res. 1069, 127-138

26 McGaraughty, S. and Heinricher, M.M. (2002) Microinjection of morphine into various amygdaloid nuclei differentially affects nociceptive responsiveness and RVM neuronal activity. Pain 96, 153-162

27 Pinto-Ribeiro, F. et al. (2006) Influence of arthritis on descending modulation of pain from the paraventricular nucleus of the hypothalamus in the rat. Acta Physiol. 186 (Suppl. 1), 121 (Pohl, U. et al., eds)

28 Han, J.S. et al. (2005) Critical role of calcitonin gene-related peptide 1 receptors in the amygdala in synaptic plasticity and pain behavior. $J$. Neurosci. 25, 10717-10728

29 Danziger, N. et al. (2001) Stage-dependent changes in the modulation of spinal nociceptive neuronal activity during the course of inflammation. Eur. J. Neurosci. 13, 230-240

30 Zambreanu, L. et al. (2005) A role for the brainstem in central sensitisation in humans. Evidence from functional magnetic resonance imaging. Pain 114, 397-407

31 Guo, W. et al. (2006) Supraspinal brain-derived neurotrophic factor signaling: a novel mechanism for descending pain facilitation. J. Neurosci. 26, 126-137

32 Gardell, L.R. et al. (2003) Enhanced evoked excitatory transmitter release in experimental neuropathy requires descending facilitation. J. Neurosci. 23, 8370-8379

33 Castro, A.R. et al. (2006) Differential expression of NK1 and GABA(B) receptors in spinal neurones projecting to antinociceptive or pronociceptive medullary centers. Brain Res. Bull. 69, 266-275

34 Neto, F.L. et al. (1999) Supraspinal metabolic activity changes in the rat during adjuvant monoarthritis. Neuroscience 94, 607-621

35 Pinto, M. et al. (2003) Noxious-evoked c-fos expression in brainstem neurons immunoreactive for GABAB, mu-opioid and NK-1 receptors. Eur. J. Neurosci. 17, 1393-1402

36 Crown, et al. (2005) Upregulation of the phosphorylated form of CREB in spinothalamic tract cells following spinal cord injury: relation to central neuropathic pain. Neurosci. Lett. 384, 139-144

37 Guan, Y. et al. (2006) Windup in dorsal horn neurons is modulated by endogenous spinal mu-opioid mechanisms. J. Neurosci. 26, 4298-4307

38 Benrath, J. et al. (2005) Low dose of S+-ketamine prevents long-term potentiation in pain pathways under strong opioid analgesia in the rat spinal cord in vivo. Br. J. Anaesth. 95, 518-523

39 Hu, X.D.etal. (2006) Diazepam inhibits the induction and maintenance of LTP of C-fiber evoked field potentials in spinal dorsal horn of rats. Neuropharmacology 50, 238-244

40 Gao, X. et al. (2005) Enhancement of NMDA receptor phosphorylation of the spinal dorsal horn and nucleus gracilis neurons in neuropathic rats. Pain 116, 62-72

41 Paxinos and Watson Atlas (1998) The rat brain in stereotaxic coordinates (4th edn), Academic Press 